\title{
Comparison of changes in nutritional status, pancreatic function and quality of life according to gender after pancreatectomy
}

\author{
Yong Chan SHIN, Sung Won JUNG*
}

Department of Surgery, Ilsan Paik Hospital, Inje University College of Medicine, Goyang, Korea

Introduction: To compare and analyze changes in nutritional status, pancreatic function and quality of life (QoL) according to gender after pancreatectomy.

Methods: Patients undergoing pancreaticoduodenectomy (PD) or distal pancreatectomy (DP) between 2007 and 2013 were included. Data on relative body weight (RBW); triceps skinfold thickness (TSFT); body mass index (BMI); serum protein, albumin, transferrin, fasting blood glucose, postprandial 2-h glucose, and stool elastase levels; and QoL questionnaire scores were collected serially for 5 years.

Results: RBW, TSFT, and BMI showed similar patterns of change between male and female patients. Biochemical parameters recovered to preoperative values 3 months after surgery in both groups. There was no significant difference in the incidence of diabetes mellitus or impaired fasting glucose between men and women during the follow-up period except for 6 months after surgery. Stool elastase levels showed similar patterns in the two groups from 12 months after surgery. Male patients scored relatively higher than female patients on the EORTC QLQ-C30 questionnaire. There was a significant difference in global health status/QoL between the two groups at 6 and 12 months after surgery and female gender was an independent risk factor for low global health status/QoL in a multivariate analysis. In EORTC QLQ-PAN26 questionnaire, female patients showed a relatively slow decrease in symptom scale scores than male patients.

Conclusions: Although female patients showed better nutritional status than males after pancreatectomy, the rate of complaining of functional difficulties and surgery-related symptoms was relatively higher than males in quality of life analysis. 